\title{
Acanthamoeba Keratitis in Egypt: Characteristics and Treatment Outcomes
}

\author{
Mohamed H Nasef \\ Sharif Y EI Emam (D) \\ Mohamed S ElShorbagy \\ Waleed A Allam \\ Ophthalmology Department, Tanta \\ University, Faculty of Medicine, Tanta, \\ Egypt
}

This article was published in the following Dove Press journal: Clinical Ophthalmology

\begin{abstract}
Purpose: To study the predisposing factors, clinical manifestations, and treatment outcome of patients with Acanthamoeba keratitis (AK) at Tanta University's Ophthalmology Hospital in Tanta, Egypt.
\end{abstract}

Methods: A retrospective study of 42 patients (44 eyes) with Acanthamoeba keratitis who had medical records available for review over 4 years.

Results: Forty-four eyes of 42 patients were treated for AK over the study period. In 29 eyes $(65.8 \%)$, AK was related to contact lens wear. Severe ocular pain was the main presenting symptom in 38 eyes $(86.3 \%)$. The most common ocular signs were radial perineural corneal infiltrates (65.9\%), pseudo-dendrites (43.2\%), ring infiltrates $(45.5 \%)$, and diffuse stromal infiltration (59\%). Acanthamoeba was detected by culture, smear, and in-vivo confocal microscopy (IVCM) in 25 eyes (56.8\%), while in 19 eyes $(43.2 \%)$ the diagnosis was based solely on the clinical findings. IVCM was effective in detection of Acanthamoeba in cases with early presentation, while culture was more sensitive in late presentation with corneal melting. The mean duration of treatment was $73.3 \pm 23.7$ days. Surgical intervention in the form of tectonic grafts or amniotic membrane transplant was required in five cases $(11.3 \%)$ due to progressive corneal thinning and perforation. Seventeen patients $(38.6 \%)$ had 0.2 or better final best-corrected visual acuity after treatment.

Conclusion: The diagnosis of AK remains a major challenge for most ophthalmologists. Contact lens abuse is the major risk factor. Early diagnosis and appropriate treatment of AK with biocidal agents can improve the final outcome and help avoid surgical intervention. IVCM is an excellent tool for early diagnosis of AK.

Keywords: acanthamoeba, keratitis, contact lenses, confocal microscopy

\section{Introduction}

Acanthamoeba keratitis (AK) is one of the most challenging cases in Ophthalmology. Misdiagnosis is not unusual, and in many instances, it presents in ways similar to keratitis of herpetic and fungal origins. ${ }^{1,2}$

The provisional diagnosis of $\mathrm{AK}$ is often based on the clinical features and invivo confocal microscopy (IVCM). However, such diagnosis is confirmed by culture, histopathologic examination, and identification by polymerase chain reaction (PCR). ${ }^{3,4}$

Acanthamoeba species are free-living amoebae, ubiquitous in the environment. Acanthamoeba is found in the air, soil, and fresh or brackish waters. The causal agent exists in both active (trophozoite) and dormant (cyst) forms. The cysts are able to survive for long periods of time in hostile environments. The trophozoites produce a variety of enzymes that aid in tissue penetration and destruction. ${ }^{5}$
Correspondence: Mohamed H Nasef Ophthalmology Department, Tanta University's Faculty of Medicine, Tanta, Egypt

Tel +201002826555

Email mohamed.nasef@med.tanta.edu.eg
Clinical Ophthalmology 2021:15 1339-1347 DovePress f in 口

Clinical Ophthalmology $2021: 151339$.

cc) (i) (5) 2021 Nasef et al. This work is published and licensed by Dove Medical Press Limited. The full terms of this license are available at https://www.dovepress.com/terms.php you hereby accept the Terms. Non-commercial uses of the work are permitted without any further permission from Dove Medical Press Limited, provided the work is properly attributed. For permission for commercial use of this work, please see paragraphs 4.2 and 5 of our Terms (https://www.dovepress.com/terms.php). 
Most of Acanthamoeba infections are associated with contact lens (CL) wearing. ${ }^{1,6}$ Infections related to CLs are often associated with inadequate care such as overuse, poor cleaning, and sleeping or swimming while wearing them. ${ }^{6,7}$

Both trophozoites and cysts can adhere to the surface of soft or rigid CLs, within minimal exposure times. ${ }^{8}$ A break in the corneal epithelium may allow them to invade into the corneal stroma. ${ }^{1}$

Corneal injury by organic material is the major risk factor for AK in rural areas. ${ }^{9-11} \mathrm{AK}$ has also been reported after invasive corneal and refractive surgeries. ${ }^{12-14}$ Acanthamoeba has been found in co-infections with fungi, viruses, chlamydia, and bacteria. ${ }^{15,16}$

In early AK, severe pain is fairly common and usually disproportionate with the clinical signs. ${ }^{1,2}$ Early signs include mild conjunctival injection together with epitheliopathy including punctate keratopathy, pseudodendrites, epithelial or subepithelial infiltrates and perineural infiltrates. Limbitis with ring infiltrates are seen in less than $20 \%$ of patients. ${ }^{1,17,18}$

Later on, ring infiltrates, marked ulceration, thinning occur, as well as a secondary sterile anterior uveitis, with hypopyon in some cases. Some patients may present with corneal edema caused by endothelial plaques or a disciform reaction. ${ }^{2,17}$ Vascularization and scleritis may occur, mainly secondary to the immunologic reaction. AK rarely progresses beyond the corneal endothelium to produce intraocular infection and endophthalmitis. ${ }^{19,20}$

This retrospective study was carried out to evaluate the predisposing factors, clinical manifestations, and treatment outcome of patients with Acanthamoeba keratitis at Tanta University's Ophthalmology Hospital in Tanta, Egypt.

\section{Patients and Methods}

This retrospective study was carried out after approval by the Institutional Review Board (IRB) and Human Research Ethics Committee at Tanta University, Faculty of Medicine, and in adherence to the tenets of the Declaration of Helsinki. The patients' data confidentiality was maintained, and, after IRB approval, a consent was not required. The medical records of 42 patients (44 eyes), who had been treated for Acanthamoeba keratitis during the period from August 2016 to August 2020, were thoroughly evaluated and analyzed.

Risk factors such as contact lens wear, corneal trauma especially by organic material, and/or previous ocular surgeries were analyzed. Similarly, symptoms such as pain, redness, diminution of vision, watering, photophobia, and/ or blepharospasm were included in the analysis. Moreover, the analysis included all findings observed during thorough ophthalmological examination of all cases, with special emphasis on corneal epithelial and stromal slit lamp signs.

The management protocol was basically the same for all cases. Once AK was suspected, corneal scraping, using either a Kimura spatula or a sterile \#15 blade, was performed under sterile conditions in the operating room to obtain an adequate specimen for testing. Patients who were under any form of topical antimicrobial therapy prior to presentation were asked to stop their medications for 24 hours before corneal scraping. The obtained specimens were subjected to both microscopic examination and culture. Microscopic examination was performed for both fresh smears using potassium hydroxide $(\mathrm{KOH})$, and Gram-stained specimens. Cultures were carried out on multiple culture media, such as blood agar, chocolate agar, Sabouraud's agar, Thioglycolate broth, and nonnutrient Escherichia coli agar. Suspected contact lenses and their solutions were cultured in the same way.

In vivo confocal microscopy (IVCM) was performed using the ConfoScan 4 (Nidek Technologies Srl, Albignasego, PD, Italy) and was considered positive for Acanthamoeba if characteristic round or ovoid dense highly refractive bodies, or doubled-walled structures denoting cysts were observed on the scans. Other findings that have been observed, in addition to the cysts, include signet rings, and bright spots.

Once the diagnosis of AK was confirmed, amebicide treatment was administered with twice-hourly (day and night) therapy with polyhexamethylene biguanide (PHMB) eye drops, prepared at the pharmacy unit at Tanta University Hospital, and $0.1 \%$ propamidine isethionate (Brolene Eye Drops ${ }^{\circledR}$, Sanofi, Guildford, United Kingdom) eye drops during the first $48 \mathrm{~h}$, followed by diurnal hourly instillation during the 3 following days. The PHMB used was begun at $0.02 \%$ concentration, and was boosted up to $0.06 \%$ if an inadequate response to therapy was observed. Topical treatment was continued every 2 $\mathrm{h}$ to complete 4 weeks, subsequently every $4 \mathrm{~h}$ to complete keratitis healing.

Supplementary treatments included a combination of Moxifloxacin $0.5 \%$ (Vigamox $^{\circledR}$, Alcon Laboratories, Inc., Fort Worth, TX. USA) or Gatifloxacin 0.3\% (Zymar $^{\circledR}$, Allergan, Inc., Irvine, CA. USA), Neosporin, and/or Voriconazole $1 \%$ eyedrops prepared at the pharmacy unit 
$\left(\right.$ Vfend $^{\circledR}$, Pfizer, Inc., New York, USA). Topical cyclopentolate $1 \%$ (Cicloplejico ${ }^{\circledR}$, Alcon Cusi, Spain) was used to relieve the associated ciliary spasm. Systemic tetracyclin or doxycycline, systemic vitamin $\mathrm{C}$, and topical antiglaucomatous agents were used as well.

Following complete epithelial healing and resolution of inflammatory signs, all patients were kept on prophylactic $0.1 \%$ propamidine isethionate eyedrops 4 times daily for one year to prevent recurrences. During the follow-up period, careful slit-lamp examination was performed, for any signs of recurrent infection, and treatment was stopped if drug toxicity or hypersensitivity was observed.

Laboratory study results of participants were divided into 2 groups according to the symptoms-to-treatment duration (STD); early and late presenters (groups A and B, respectively). Group A included 23 patients ( 24 eyes) who presented within 3 weeks from the onset of symptoms, while group B included 19 patients (20 eyes) who presented after 3 weeks.

Analyses were performed by SPSS statistics for Windows, version 23.0 (IBM Corp., Armonk, NY). Quantitative data were expressed as mean \pm standard deviation (SD). Qualitative data were expressed as percentage. Independent sample $t$-test, Chi-square test, and Pearson's correlation coefficient test were used. A $p$ value of 0.05 or less was considered statistically significant.

\section{Results}

Medical records of 57 patients were assessed for study eligibility between August 2016 and August 2020, of whom 15 patients were excluded due to incomplete data. The remaining 42 cases (44 eyes) were included in the study. They were diagnosed with AK and full management was carried out at Tanta University's Ophthalmology Hospital in Tanta, Egypt.

The mean age of the participants was $31.6 \pm 11.7$ years (range from 18 to 65 years). Among the 42 cases included in the study, $30(71.4 \%)$ were females, and 12 (28.5\%) were males. Contact lens (CL) wear was the most common associated factor recorded in 34 eyes $(77 \%)$, followed by ocular trauma in 10 eyes $(23 \%)$.

Severe ocular pain was the main presenting symptom in 38 eyes $(86 \%)$. All patients complained of variable degrees of red eyes, watering, and diminution of vision. Common signs included perineural radial infiltrates in 29 eyes $(65.9 \%)$, diffuse stromal infiltrates in 26 eyes (59.1\%), ring infiltrates in 20 eyes $(45.5 \%)$, and pseudodendrites in 19 eyes (43.2\%). Less common signs such as satellite infiltrates, anterior chamber reaction, and associated scleritis were found in 11 eyes (25\%), 7 eyes $(15.9 \%)$, and 3 eyes (6.8\%) respectively (Figure $1 \mathrm{~A}-\mathrm{E})$.

The mean STD was $20.2 \pm 12.6$ days (range from 1 to 40 days). History taking revealed that up to two-thirds of the eyes had received different topical medications prescribed by other physicians prior to presentation (29 eyes (66\%) antibiotics, 17 eyes (38.6\%) antifungal, 11 eyes (25\%) corticosteroids, and 12 eyes (27.3\%) antiviral agents). Most eyes responded well to a $0.02 \%$ concentration of PHMB (40 eyes (91\%), with only 4 eyes needing a higher concentration of $0.06 \%(9 \%)$ ).

IVCM was highly sensitive in diagnosis of AK during the early stages of the disease. Acanthamoeba cysts and trophozoites were imaged in 14 eyes (58.3\%) in group A compared to 3 eyes (15\%) in group B. Similarly, smear examination revealed positive results in 12 eyes (50\%) in group A and 4 eyes (20\%) in group B. On the other hand, culture results were positive for Acanthamoeba in a higher percentage in group B (9 eyes, $45.0 \%$ ) than in group A (9 eyes, 37.5\%) (Table 1).

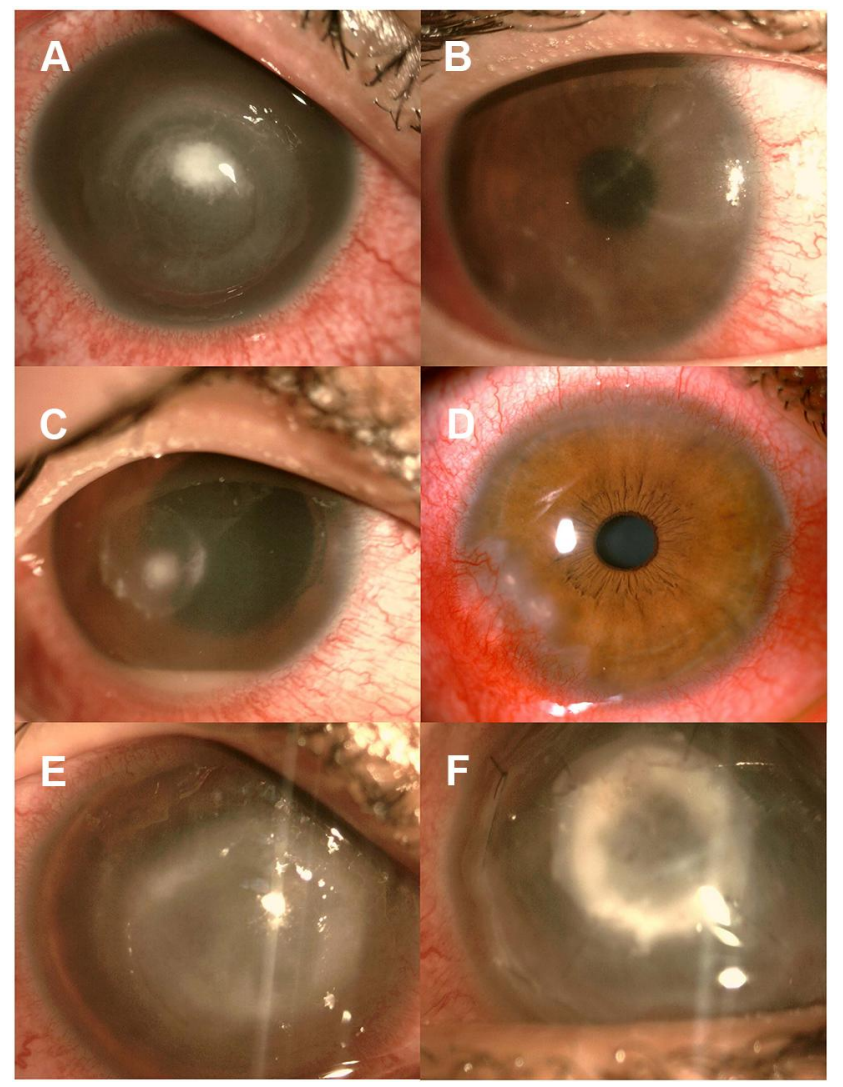

Figure I Ocular findings at presentation: Ring infiltrates ((A), 20 eyes), perineural infiltrates ((B), 29 eyes), hypopyon ((C), 7 eyes), scleritis ((D), 3 eyes), diffuse stromal infiltrates ((E), 26 eyes). After amniotic membrane grafting (F). 
Table I Results of Diagnostic Tools in Both Groups: In Group A (Early Presenters), There Was No Significant Difference Between Different Methods to Diagnose Positive Cases. However, in Group B (Late Presenters), Culture Was Significantly More Accurate in Diagnosing AK

\begin{tabular}{|l|l|l|l|l|l|}
\hline Positive Cases Diagnosed & Culture & Smear & IVCM & \multicolumn{2}{|c|}{ P } \\
\hline Group A $(n=24)$ & & & $14(58.3 \%)$ & 2.347 & 0.309 \\
\hline Group B (n=20) & $9(37.5 \%)$ & $12(50 \%)$ & $3(15 \%)$ & 4.292 & $0.038^{2}$ \\
\hline & $9(45 \%)$ & $4(20 \%)$ & $0.033^{*}$ & & \\
\hline
\end{tabular}

Note: Asterisk $(*)$ indicates statistical significance.

IVCM, smear examination, and cultures revealed no statistically significant differences among them as tools for diagnosis of AK during the early stages of the disease. However, culture was more sensitive as a diagnostic tool at late stages (Table 1).

The mean duration of treatment, till complete epithelial healing and resolution of the inflammatory signs, was 73.3 \pm 23.7 days (range from 32 to 130 days).

There was a strong positive correlation between the treatment duration and the STD on one hand (Figure 2), and a strong negative correlation with $\mathrm{CL}$ wear on the other (Table 2). However, no statistical correlation was found when comparing the treatment duration and final visual outcome on one hand, and between age, gender, or the use of other medications prior to presentation on the other hand. Recurrent lesions were not recorded throughout the study period.

Adjunctive surgery was required in 5 eyes (11.3\%) due to uncontrolled progressive corneal thinning and inflammation. Four eyes (9\%) received amniotic membrane

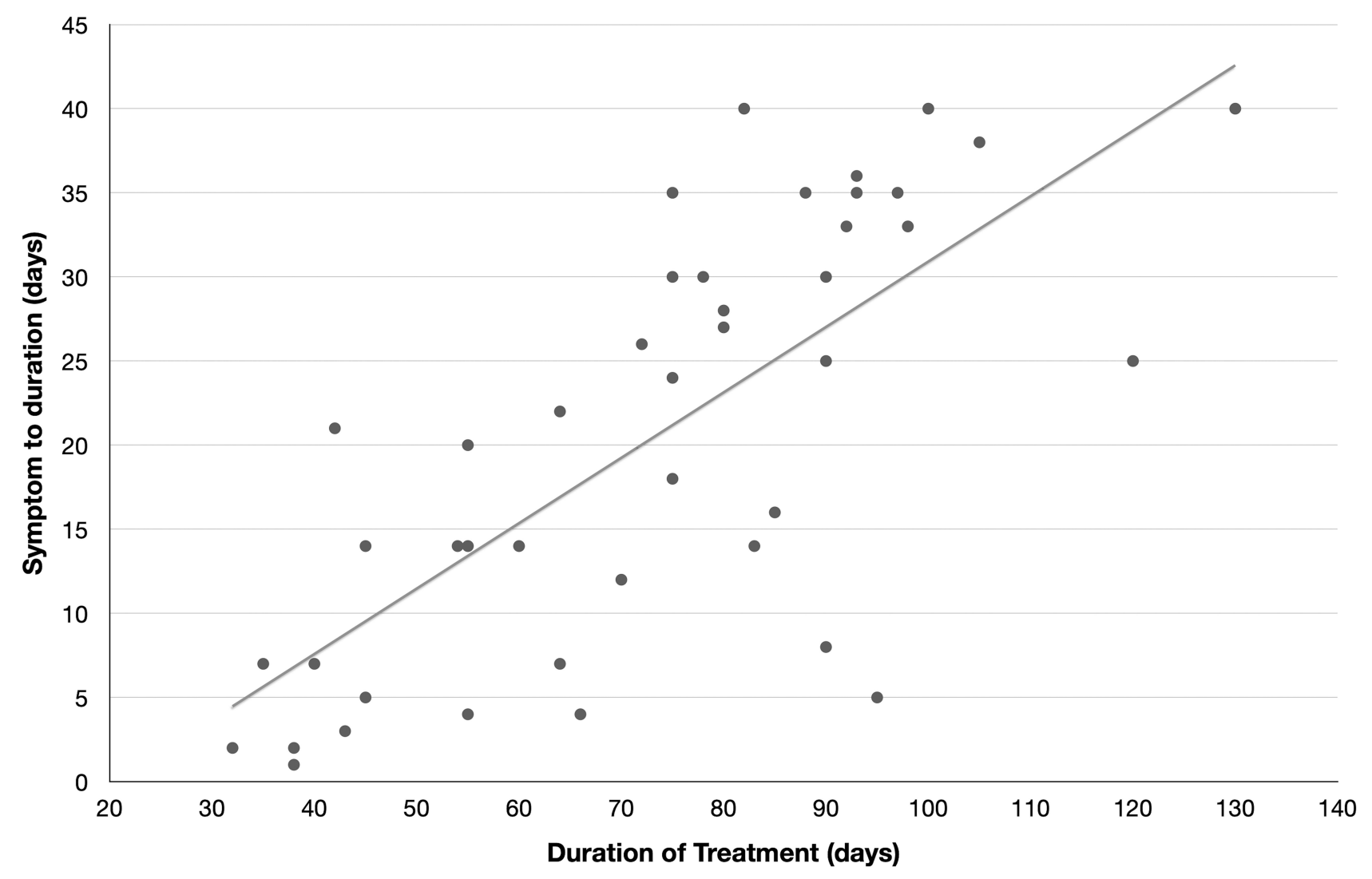

Figure 2 Correlation between symptom to duration (STD) and duration of treatment: A statistically significant positive correlation was found $(r=0.753, p=0.00 \mathrm{I})$ with linear trendline shown. 
Table 2 Correlation Between CL Wear and Treatment Duration: A Significant Negative Correlation Was Found

\begin{tabular}{|c|c|c|c|c|c|}
\hline \multicolumn{2}{|c|}{} & \multicolumn{2}{|c|}{ Treatment Duration (Days) } & \multicolumn{2}{c|}{} \\
\cline { 2 - 6 } \multicolumn{2}{|c|}{} & Range & Mean \pm SD & \multirow{2}{*}{-Test } & p-value \\
\hline \multirow{2}{*}{ CLs } & + ve & $32-106$ & $65.76 \pm 22.34$ & 10.721 & $0.002^{*}$ \\
\cline { 2 - 4 } & -ve & $55-130$ & $88.07 \pm 19.45$ & & \\
\hline
\end{tabular}

Note: Asterisk (*) indicates statistical significance.

transplantation (AMT, Figure 1F), and one eye $(2.2 \%)$ received tectonic keratoplasty. Throughout the duration of the study, none of the 44 eyes included lost one line or more of uncorrected distant visual acuity (UDVA), compared to the baseline UDVA. The final visual acuity outcome is illustrated in Figure 3.

\section{Discussion}

In the current study, we observed a higher incidence of AK among females. A finding that may be attributed to the higher prevalence of CL wear among them. ${ }^{1,21}$ Similar to other studies, ${ }^{22,23} \mathrm{CL}$ wear with bad hygiene was the most common risk factor of $\mathrm{AK}$ in our series. Based on the rural nature of the community where our study was performed, trauma by organic material was recorded as the second most common risk factor of $\mathrm{AK}$ due to prevalence of agricultural activities among the population.
The definitive diagnosis of $\mathrm{AK}$ should be based on positive results of imaging and/or laboratory tests. However, in our series, cases with negative results received the treatment of $\mathrm{AK}$ based on the history and evident clinical findings. Many studies have reported the clinical signs associated with AK, summarized in Table $3 .^{24-33}$ In our study, perineural radial infiltrates were the most commonly encountered sign followed by diffuse stromal and ring infiltrates, and pseudodendrites. Other less frequent signs included satellite infiltrates, anterior chamber reaction, and associated scleritis. Such a wide variety is attributed to the age and general condition of the patient, severity of infection, STD, and the medications administered prior to presentation.

IVCM is a noninvasive method through which Acanthamoeba cysts can be immediately detected especially in early presenting cases. ${ }^{34}$ Studies using IVCM have found a high sensitivity of $100 \%$ and $84 \%$ specificity for the diagnosing $\mathrm{AK}$, compared to microbiological studies of corneal scrapings. ${ }^{35,36}$ In our study, IVCM was successful in detection of the cysts in $58.3 \%$ of early presenters. However, this number decreased to $15 \%$ in cases in patients with late presentation. This difference is due to difficult visualization and identification of the cysts due to the marked reduction of contrast caused by dense infiltrates and superficial plaques in advanced cases.

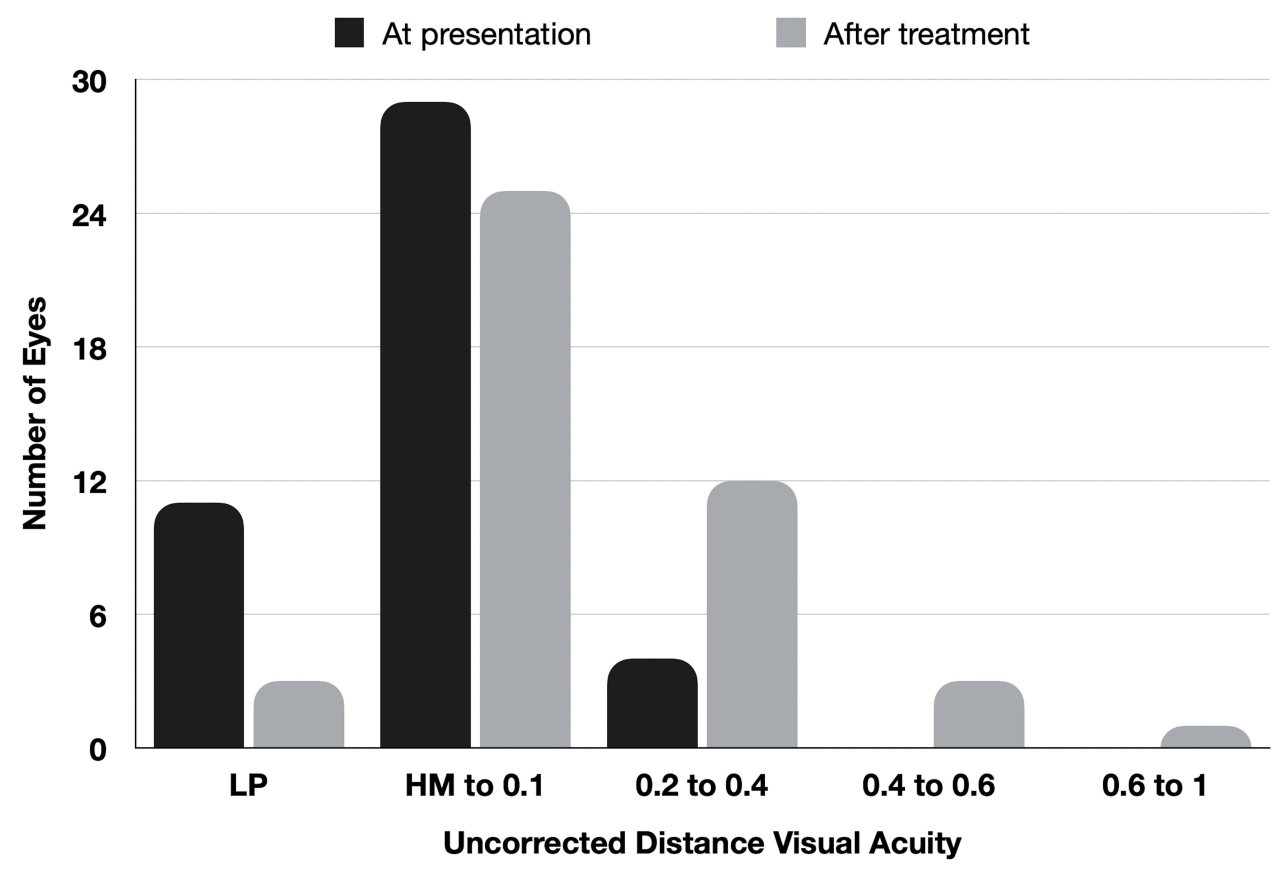

Figure 3 Uncorrected distance visual acuity: at presentation, and after treatment. 


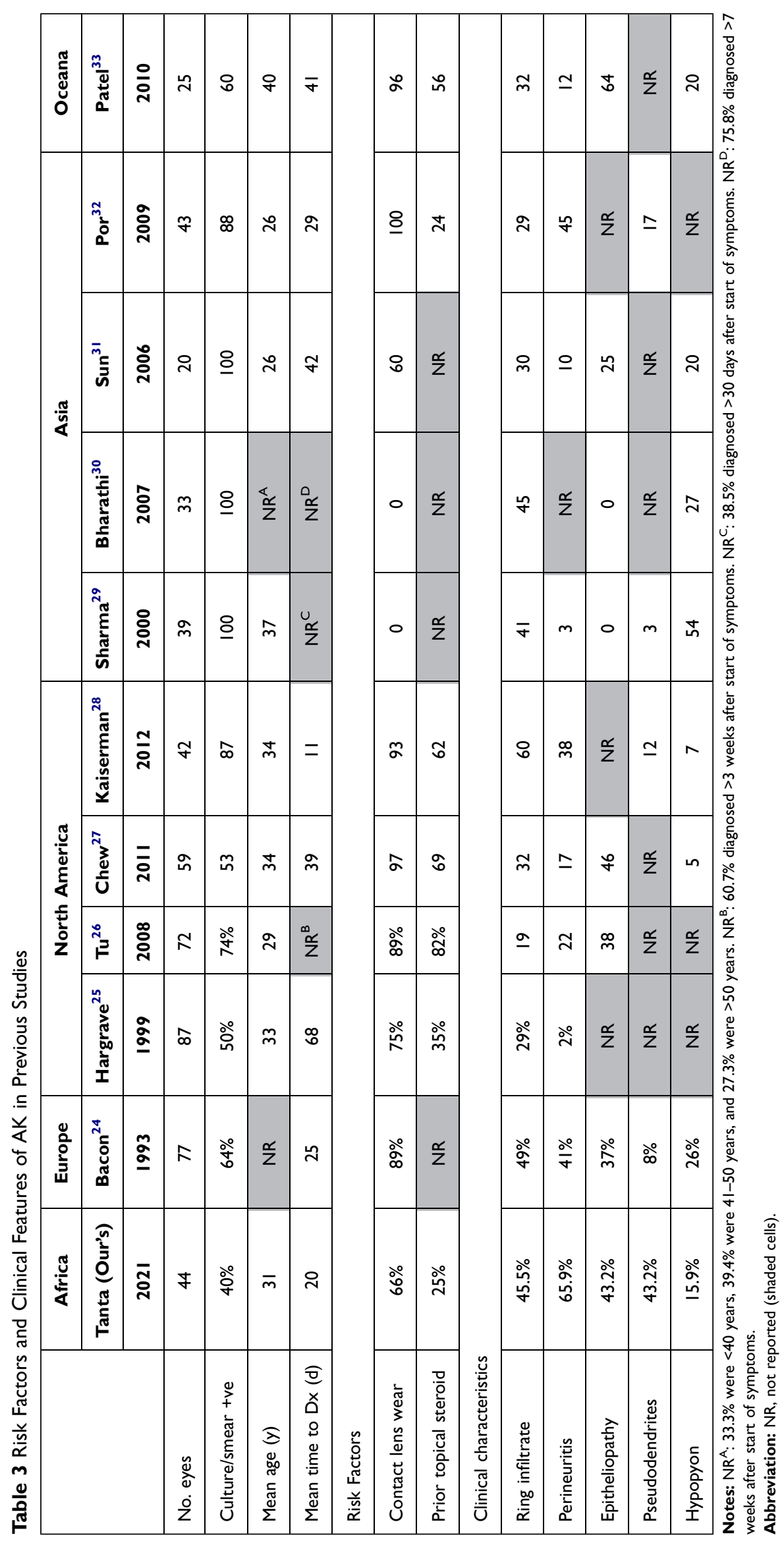


The sensitivity of microbial cultures and smear examination for diagnosis of $\mathrm{AK}$ is affected by many factors, such as prior administration of antimicrobial agents, adequate sampling, and the need for special growth media. A great variability is seen in previous studies, that have reported positive cultures as low as $7 \%$, and as high as $57 \%$ of cases with clinical signs suggesting $\mathrm{AK}^{4,37}$ In 2008, Tu et al, concluded that cultures are more helpful in diagnosis of $\mathrm{AK}$ in late presenters compared to early ones. ${ }^{38}$ A conclusion that was confirmed by our results, where $37 \%$ of cultures revealed positive results during the early stages of $\mathrm{AK}$ compares to $45 \%$ of cases with advanced disease. However, the difference between both groups was not statistically significant.

Pathological examination with direct visualization of Acanthamoeba using light microscope, via simultaneous testing of smear and superficial corneal scraping, has been reported to result in a sensitivity of up to $83.0 \%$, in patients with clinical evidence of $\mathrm{AK} .{ }^{38}$ In our series, the sensitivity of smear examination, compared to clinical diagnosis, was $50 \%$ in the early group, however this decreased to $20 \%$ in the late group. Amoebae density is usually low, in patients pre-treated with antibiotics.

It is fully understood that early diagnosis and treatment of $\mathrm{AK}$ is associated with better prognosis and lesser need for surgical intervention. ${ }^{39}$ Currently, diamidines and biguanides are the most common cysticidal agents used for treatment of AK. Multiple studies have shown that they have the best and most constant cysticidal and amoebicidal activities. ${ }^{40-42}$ We used a combination of topical propamidine isethionate $0.1 \%$ and PHMB $0.02 \%$ in most cases. The concentration of PHMB was increased in 4 eyes only, when poor response was observed. After complete resolution of disease activity and epithelial healing, prophylactic topical propamidine isethionate $0.1 \%$ was used for one year to ensure long-term treatment efficacy and to avoid relapses. A 6-12-month duration of treatment has been advised by some authors. ${ }^{43}$ Moreover, close observation of our patients in the follow-up period was performed to detect early signs of toxicity, or recurrence if therapy was stopped. Topical amoebicidal drugs are known to be toxic to human corneal cells; fortunately, minimal concentrations are effective and not toxic to corneal epithelial cells. $^{44}$

In our study, the mean treatment duration was $73.3 \pm$ 23.7 days. Late presentation, deep penetration and encystment of the organism and poor penetration of the biocides are all associated with longer treatment courses.
In conclusion, the diagnosis of $\mathrm{AK}$ remains a major challenge for most ophthalmologists. Contact lens abuse is the major risk factor. Early diagnosis and appropriate treatment of AK with biocidal agents can improve the final outcome and help avoid surgical intervention. IVCM is an excellent tool for early diagnosis of AK.

To the best of our knowledge, this is one of the largest studies with long follow-up assessing the diagnosis and management of acanthamoeba keratitis. Lack of polymerase chain reaction (PCR) testing, a technology not available in our facility, is a major limitation to this study.

\section{Disclosure}

The authors report no conflicts of interest for this work.

\section{References}

1. Maycock NJ, Jayaswal R. Update on acanthamoeba keratitis: diagnosis, treatment, and outcomes. Cornea. 2016;35:713-720. doi:10.1097/ICO.0000000000000804

2. Page MA, Mathers WD. Acanthamoeba keratitis: a 12-year experience covering a wide spectrum of presentations, diagnoses, and outcomes. J Ophthalmol. 2013;2013:670242. doi:10.1155/2013/ 670242

3. Ikeda Y, Miyazaki D, Yakura K, et al. Assessment of real-time polymerase chain reaction detection of Acanthamoeba and prognosis determinants of Acanthamoeba keratitis. Ophthalmology. 2012;119:1111-1119. doi:10.1016/j.ophtha.2011.12.023

4. Dart JK, Saw VP, Kilvington S. Acanthamoeba keratitis: diagnosis and treatment update 2009. Am J Ophthalmol. 2009;148:487-499 e2. doi:10.1016/j.ajo.2009.06.009

5. Siddiqui R, Khan NA. Biology and pathogenesis of Acanthamoeba. Parasit Vectors. 2012;5:6. doi:10.1186/1756-3305-5-6

6. Pacella E, La Torre G, De Giusti M, et al. Results of case-control studies support the association between contact lens use and Acanthamoeba keratitis. Clin Ophthalmol. 2013;7:991-994. doi: 10.2147/OPTH.S43471

7. Joslin CE, Tu EY, Shoff ME, et al. The association of contact lens solution use and Acanthamoeba keratitis. Am J Ophthalmol. 2007;144:169-180. doi:10.1016/j.ajo.2007.05.029

8. Sharma S, Ramachandran L, Rao GN. Adherence of cysts and trophozoites of Acanthamoeba to unworn rigid gas permeable and soft contact lenses. CLAO J. 1995;21:247-251.

9. Pan XJ, Jiang T, Zhu H, Liu PP, Zhou ZY, Mao AJ. Corneal infection in Shandong peninsula of China: a 10-year retrospective study on 578 cases. Int J Ophthalmol. 2016;9:53-57. doi:10.18240/ijo.2016.01.09

10. Lalitha P, Lin CC, Srinivasan M, et al. Acanthamoeba keratitis in South India: a longitudinal analysis of epidemics. Ophthalmic Epidemiol. 2012;19:111-115. doi:10.3109/09286586.2011.645990

11. Mascarenhas J, Lalitha P, Prajna NV, et al. Acanthamoeba, fungal, and bacterial keratitis: a comparison of risk factors and clinical features. Am $J$ Ophthalmol. 2014;157:56-62. doi:10.1016/j. ajo.2013.08.032

12. Arnalich-Montiel F, Almendral A, Arnalich F, Valladares B, LorenzoMorales J. Mixed Acanthamoeba and multidrug-resistant Achromobacter xyloxidans in late-onset keratitis after laser in situ keratomileusis. J Cataract Refract Surg. 2012;38:1853-1856. doi:10.1016/j.jcrs.2012.08.022

13. Garg P, Chaurasia S, Vaddavalli PK, Muralidhar R, Mittal V, Gopinathan U. Microbial keratitis after LASIK. J Refract Surg. 2010;26:209-216. doi:10.3928/1081597X-20100224-07 
14. Sharma DP, Sharma S, Wilkins MR. Microbial keratitis after corneal laser refractive surgery. Future Microbiol. 2011;6:819-831. doi: $10.2217 / \mathrm{fmb} .11 .61$

15. Lin HC, Hsiao CH, Ma DH, et al. Medical treatment for combined Fusarium and Acanthamoeba keratitis. Acta Ophthalmol. 2009;87:199-203. doi:10.1111/j.1755-3768.2008.01192.x

16. Tu EY, Joslin CE, Nijm LM, Feder RS, Jain S, Shoff ME. Polymicrobial keratitis: Acanthamoeba and infectious crystalline keratopathy. Am J Ophthalmol. 2009;148:13-9 e2. doi:10.1016/j. ajo.2009.01.020

17. Ross J, Roy SL, Mathers WD, et al. Clinical characteristics of Acanthamoeba keratitis infections in 28 states, 2008 to 2011. Cornea. 2014;33:161-168. doi:10.1097/ICO.0000000000000014

18. Iovieno A, Gore DM, Carnt N, Dart JK. Acanthamoeba sclerokeratitis: epidemiology, clinical features, and treatment outcomes. Ophthalmology. 2014;121:2340-2347. doi:10.1016/j. ophtha.2014.06.033

19. Rammohan R, Hajib Naraharirao M, Veerappan S, et al. Cluster of post-operative endophthalmitis caused by Acanthamoeba T10 genotype - a first report. Cornea. 2021;40:232-241. doi:10.1097/ ICO.0000000000002603

20. Kowalski RP, Nayyar SV, Romanowski EG, et al. The prevalence of bacteria, fungi, viruses, and Acanthamoeba from 3004 cases of keratitis, endophthalmitis, and conjunctivitis. Eye Contact Lens. 2020;46:265-268. doi:10.1097/ICL.0000000000000642

21. Chin J, Young AL, Hui M, Jhanji V. Acanthamoeba keratitis: 10-year study at a tertiary eye care center in Hong Kong. Cont Lens Anterior Eye. 2015;38:99-103. doi:10.1016/j.clae.2014.11.146

22. Beattie TK, Tomlinson A, Seal DV. Surface treatment or material characteristic: the reason for the high level of Acanthamoeba attachment to silicone hydrogel contact lenses. Eye Contact Lens. 2003;29: SS40-SS44. doi:10.1097/00140068-200301001-00012

23. Jo YJ, Jang SK, Lee J, Lee JS. A 5-year review of acanthamoeba keratitis related to wearing contact lenses in Korea. Eye Contact Lens. 2020;46:223-227. doi:10.1097/ICL.0000000000000669

24. Bacon AS, Frazer DG, Dart JK, Matheson M, Ficker LA, Wright P. A review of 72 consecutive cases of Acanthamoeba keratitis, 1984-1992. Eye (Lond). 1993;7(6):719-725. doi:10.1038/ eye. 1993.168

25. Hargrave SL, McCulley JP, Husseini Z. Results of a trial of combined propamidine isethionate and neomycin therapy for Acanthamoeba keratitis. Brolene Study Group. Ophthalmology. 1999;106:952-957. doi:10.1016/S0161-6420(99)00515-1

26. Tu EY, Joslin CE, Sugar J, Shoff ME, Booton GC. Prognostic factors affecting visual outcome in Acanthamoeba keratitis. Ophthalmology. 2008;115:1998-2003. doi:10.1016/j.ophtha.2008.04.038

27. Chew HF, Yildiz EH, Hammersmith KM, et al. Clinical outcomes and prognostic factors associated with acanthamoeba keratitis. Cornea. 2011;30:435-441. doi:10.1097/ICO.0b013e3181ec905f

28. Kaiserman I, Bahar I, McAllum P, et al. Prognostic factors in Acanthamoeba keratitis. Can J Ophthalmol. 2012;47:312-317. doi:10.1016/j.jcjo.2012.03.040

29. Sharma S, Garg P, Rao GN. Patient characteristics, diagnosis, and treatment of non-contact lens related Acanthamoeba keratitis. $\mathrm{Br}$ J Ophthalmol. 2000;84:1103-1108. doi:10.1136/bjo.84.10.1103

30. Bharathi JM, Srinivasan M, Ramakrishnan R, Meenakshi R, Padmavathy S, Lalitha PN. A study of the spectrum of Acanthamoeba keratitis: a three-year study at a tertiary eye care referral center in South India. Indian J Ophthalmol. 2007;55:37-42. doi:10.4103/0301-4738.29493
31. Sun X, Zhang Y, Li R, et al. Acanthamoeba keratitis: clinical characteristics and management. Ophthalmology. 2006;113:412-416. doi:10.1016/j.ophtha.2005.10.041

32. Por YM, Mehta JS, Chua JL, et al. Acanthamoeba keratitis associated with contact lens wear in Singapore. Am J Ophthalmol. 2009;148:712 e2. doi:10.1016/j.ajo.2009.02.030

33. Patel DV, Rayner S, McGhee CN. Resurgence of Acanthamoeba keratitis in Auckland, New Zealand: a 7-year review of presentation and outcomes. Clin Exp Ophthalmol. 2010;38:15-20; quiz 87. doi:10.1111/j.1442-9071.2009.02182.x

34. Li S, Bian J, Wang Y, Wang S, Wang X, Shi W. Clinical features and serial changes of Acanthamoeba keratitis: an in vivo confocal microscopy study. Eye (Lond). 2020;34:327-334. doi:10.1038/s41433-0190482-3

35. Kanavi MR, Javadi M, Yazdani S, Mirdehghanm S. Sensitivity and specificity of confocal scan in the diagnosis of infectious keratitis. Cornea. 2007;26:782-786. doi:10.1097/ICO.0b013e318064582d

36. Labbe A, Khammari C, Dupas B, et al. Contribution of in vivo confocal microscopy to the diagnosis and management of infectious keratitis. Ocul Surf. 2009;7:41-52. doi:10.1016/S1542-0124(12) 70291-4

37. Yera H, Zamfir O, Bourcier T, et al. Comparison of PCR, microscopic examination and culture for the early diagnosis and characterization of Acanthamoeba isolates from ocular infections. Eur J Clin Microbiol Infect Dis. 2007;26:221-224. doi:10.1007/s10096-0070268-6

38. Tu EY, Joslin CE, Sugar J, Booton GC, Shoff ME, Fuerst PA. The relative value of confocal microscopy and superficial corneal scrapings in the diagnosis of Acanthamoeba keratitis. Cornea. 2008;27:764-772. doi:10.1097/ICO.0b013e31816f27bf

39. Claerhout I, Goegebuer A, Van Den Broecke C, Kestelyn P. Delay in diagnosis and outcome of Acanthamoeba keratitis. Graefes Arch Clin Exp Ophthalmol. 2004;242:648-653. doi:10.1007/s00417-003-08057

40. Bagga B, Sharma S, Gour RP, et al. A randomized masked pilot clinical trial to compare the efficacy of topical $1 \%$ voriconazole ophthalmic solution as monotherapy with combination therapy of topical $0.02 \%$ polyhexamethylene biguanide and $0.02 \%$ chlorhexidine in the treatment of Acanthamoeba keratitis. Eye (Lond). 2020. doi:10.1038/s41433-020-1109-4

41. Lim N, Goh D, Bunce C, et al. Comparison of polyhexamethylene biguanide and chlorhexidine as monotherapy agents in the treatment of Acanthamoeba keratitis. Am J Ophthalmol. 2008;145:130-135. doi:10.1016/j.ajo.2007.08.040

42. Duguid IG, Dart JK, Morlet N, et al. Outcome of acanthamoeba keratitis treated with polyhexamethyl biguanide and propamidine. Ophthalmology. 1997;104:1587-1592. doi:10.1016/S0161-6420(97) 30092-X

43. Lorenzo-Morales J, Khan NA, Walochnik J. An update on Acanthamoeba keratitis: diagnosis, pathogenesis and treatment. Parasite. 2015;22:10. doi:10.1051/parasite/2015010

44. Carrijo-Carvalho LC, Sant'ana VP, Foronda AS, de Freitas D, de Souza Carvalho FR. Therapeutic agents and biocides for ocular infections by free-living amoebae of Acanthamoeba genus. Surv Ophthalmol. 2017;62(2):203-218. doi:10.1016/j.survophthal. 2016.10.009 


\section{Publish your work in this journal}

Clinical Ophthalmology is an international, peer-reviewed journal covering all subspecialties within ophthalmology. Key topics include: Optometry; Visual science; Pharmacology and drug therapy in eye diseases; Basic Sciences; Primary and Secondary eye care; Patient Safety and Quality of Care Improvements. This journal is indexed on PubMed
Central and CAS, and is the official journal of The Society of Clinical Ophthalmology (SCO). The manuscript management system is completely online and includes a very quick and fair peer-review system, which is all easy to use. Visit http://www.dovepress.com/ testimonials.php to read real quotes from published authors.

Submit your manuscript here: https://www.dovepress.com/clinical-ophthalmology-journal 